\title{
ENTORNO, CENTROS Y COMUNIDAD DE ESCUELAS RURALES DEL NORTE (CANTABRIA) Y SUR DE ESPAÑA (HUELVA)
}

\author{
Carmen Álvarez Álvarez \\ Universidad de Cantabria \\ Francisco Javier García-Prieto \\ Francisco J. Pozuelos-Estrada \\ Universidad de Huelva
}

RESUMEN: Las escuelas rurales en España constituyen una realidad tan amplia y multiforme como invisible y compleja, sin embargo, no contamos con estudios comparativos entre regiones que muestren las diferentes realidades que existen bajo el término "escuela rural" en nuestro país. Por ello, en este artículo nos preguntamos por las semejanzas y diferencias que existen entre las escuelas rurales del norte y del sur de España. En este sentido, se desarrolla un estudio cuantitativo y cualitativo en el que se explora la situación de las escuelas rurales mediante un cuestionario y entrevistas en profundidad en tres tipos de centros (aulas unitarias, centros rurales agrupados y centros graduados ubicados en el medio rural) en dos provincias muy rurales tradicionalmente: Cantabria y Huelva. Se observan contrastes en el entorno y el profesorado, y similitudes en los centros, el alumnado y las familias. Las escuelas están menos aisladas y cuentan con un profesorado más estable en el norte. El profesorado se muestra optimista y cuenta con un alumnado bien dispuesto. La participación de las familias es mejorable, pero tienen altas expectativas en la escuela. El reto es y sigue siendo asegurar un sistema educativo equitativo que ayude a mantener vivo el mundo rural.

PALABRAS CLAVE: escuela rural, zona rural, relación Escuela-comunidad, escuela unitaria, análisis comparativo.

\section{ENVIRONMENT, CENTERS AND COMMUNITY OF RURAL SCHOOLS OF THE NORTH (CANTABRIA) AND SOUTH (HUELVA) OF SPAIN}

ABSTRACT: Rural schools in Spain constitute a reality as wide and multiform as invisible and complex; however, we do not have comparative studies between regions that show the different realities that exist under the term "rural 
school" in our country. Therefore, in this paper we ask ourselves the similarities and differences that exist between the rural schools of the north and the south of Spain. In this sense, a quantitative and qualitative study is developed in which the situation of rural schools is explored by means of a questionnaire and in-depth interviews in three types of centres (unitary classrooms, grouped rural centres and graduate centres located in the rural environment) in two traditionally very rural provinces: Cantabria and Huelva. Contrasts are observed in the setting and the teaching staff and similarities in the centres, the students and the families. The schools are less isolated and have a more stable teaching staff in the north. The teachers are optimistic and have a well-disposed student body. The participation of families can be improved, but they have high expectations of the school. The challenge is and remains to ensure an equitable education system that helps keep the rural world alive.

KEYWORDS: Rural school, rural area, school-community relationship, one teacher school, comparative analysis.

Recibido: 26/03/2020

Aceptado: 02/05/2020

Correspondencia: Carmen Álvarez Álvarez. Facultad de Educación, Universidad de Cantabria, Avda. de los Castros, s/n, 39005 Santander. Email: carmen.alvarez@unican.es.

\section{Marco teórico}

Con el término "escuela rural" en España se pueden designar realidades escolares muy diversas y complejas extendidas a lo largo y ancho de la geografía. Pese a esto, las escuelas rurales constituyen una realidad relevante, amplia y multiforme frecuentemente invisible porque el modelo de escuela urbana se ha ido imponiendo con fuerza (Heredero, Collado-González y Shoiti-Nozu, 2016; Sepúlveda-Ruiz y Gallardo-Gil, 2011; Vázquez-Recio, 2016). Esta situación afecta notablemente a la investigación escolar, siendo más fácil encontrar resultados de estudios realizados en escuelas urbanas que en centros rurales (Abós, 2015; Barthes, Champollion y Alpe, 2018; Bustos, 2011). Revisiones como la de Santamaría (2018) destacan algunas comparativas rurales-urbanas o "brechas" como indica el autor, respecto a la formación docente, abandono escolar, nivel educativo de la población o evaluaciones, entre otras. Otros estudios más específicos como el de García-Valcárcel, Basilotta, y Mulas (2016) contrastan igualmente la escuela rural con la urbana. No obstante, los estudios empíricos se suelen localizar en regiones concretas como el realizado por Burrial, Sala, Samper y Torres (2018) evaluando diversas variables de la educación rural catalana. Y son circunstanciales o escasos a nivel del territorio nacional, realizándose escasos estudios comparativos que nos muestren las diferentes realidades de las escuelas rurales entre regiones alejadas (Hamodi y AraguésGarde, 2014; Pérez, García y Quijano, 2018). Una de estas investigaciones en territorios distintos es la de Abós, Boix, Bustos, Domingo, Domingo, y Ramo (2015) 
que se realiza a nivel de Comunidades Autónomas (Andalucía, Aragón y Cataluña). Por ello, consideramos relevante realizar un estudio comparativo entre dos grandes zonas en las que prevemos que pueden darse contrastes en su desarrollo escolar: Cantabria en el norte y Huelva en el sur con la intención de cotejar el estado de variables relevantes en la diversa tipología de centros rurales existentes: CEIPS, aulas unitarias, Centros rurales agrupados (CRA)/Colegios públicos rurales (CPR) y Centros Rurales de Innovación Educativa. Ambas provincias tradicionalmente han sido muy rurales.

Una vez identificados los diferentes tipos de escuelas es necesario acotar brevemente cómo es la sociedad rural española actual, el profesorado, el alumnado, las familias, etc., ya que sobre estos elementos centraremos el foco en el presente trabajo empírico, procurando aumentar el conocimiento científico sobre la materia. Nuestra hipótesis de partida es que habrá semejanzas y diferencias entre el norte (Cantabria) y el sur (Huelva) de nuestro país en cuanto a entorno, centros, profesorado, alumnado y familias.

Desde hace algunos años España, como muchos otros países, se encuentra en un proceso de tránsito de una sociedad que fue mayoritariamente rural a una sociedad urbana, que nos sitúa en un tiempo plagado de contradicciones en el que cualquier intento de generalización es difícil y está lleno de matices. Cada vez el territorio rural es más diverso (en sus espacios, sociedad y cultura) y prima la heterogeneidad en las actividades productivas, la conectividad, las realidades territoriales y sociales, etc. Además, se ha producido el descenso del sector primario, el aumento del turismo y ocio rural, la proliferación de segundas residencias para el fin de semana o veraneo, el surgimiento de colectivos neorrurales, etc. dando paso a la existencia de áreas rurales dinámicas, estáticas y en decadencia. La escuela rural, como es lógico, se ve fuertemente sacudida y perjudicada por esta situación (Vázquez-Recio, 2016), lo que no impide que en otros estudios se hable de modelo de éxito (Tahull y Montero, 2018). Cada vez más las zonas rurales han ido diversificando sus actividades productivas, hablándose de "nuevas ruralidades" (Martínez y Bustos, 2011) y los núcleos más pequeños han ido perdiendo población y reduciéndose dándose un movimiento migratorio hacia las ciudades o áreas dotadas de más y mejores servicios (Gallardo, 2011). No obstante, "las diferentes comunidades autónomas presentan diferencias, en ocasiones apreciables, en cuanto a la distribución de la población y a su grado de ruralidad" (Ministerio de Educación, Cultura y Deporte-Consejo Escolar del Estado, 2015). Según estudios recientes sobre educación rural "los núcleos de población de menos de 2000 habitantes son los más proclives a perder población que los de más volumen de población" (Bustos, 2011, p. 68). Esto ha generado el cierre de una parte de las escuelas rurales y la agrupación en torno a CRAs de otros centros que han ido perdiendo alumnado. Analizando los datos del Consejo Escolar de Estado en los cursos 1999-2000 y comparándolos con los del 2007-2008 se ha concluido que "las comunidades de Andalucía, Castilla la Mancha, Cataluña, Galicia y La Rioja son las únicas que han incrementado el número de centros rurales agrupados, unidades y alumnado" (Bustos, 2011, p. 69). Si nos fijamos en las localidades donde hay CRAs se observa que "sólo se ha producido un incremento en tres comunidades: Andalucía $(+12 \%)$, Castilla la Mancha (+14\%) y Galicia (+155) [...] llevándose Cantabria (-56\%) 
y Asturias (-40\%) la peor parte" (Bustos, 2011, p. 69). Aquí encontramos una primera diferencia significativa entre regiones.

Por otro lado, fruto de este tránsito social de una sociedad rural a una urbana, las escuelas situadas en entornos rurales atraviesan un proceso de hibridación en el que conviven en relativa armonía elementos escolares rurales y urbanos simultáneamente (Benito, 2013). Por citar dos ejemplos, puede señalarse que (1) la multigraduación es perfectamente compatible con el empleo de libros de texto y (2) la convivencia escolar de un reducido grupo de alumnos con carácter familiar no impide una enseñanza orientada a un academicismo con pocas relaciones interpersonales (Barthes, Champollion y Alpe, 2018). Todo parece indicar que la escuela rural acusa los efectos de la globalización, pero no sabemos si lo hace en el mismo grado en las diferentes regiones de España y si al hacerlo genera fuertes choques culturales (Sepúlveda-Ruiz y Gallardo-Gil, 2011).

En relación al profesorado, la investigación educativa ha venido poniendo de manifiesto una predilección por los centros urbanos o ubicados en zonas de mayor densidad de población, viviendo con mayor anonimato del que se tiene en un pueblo (Sánchez, Martínez y Lucena, 2017). Los docentes, con demasiada frecuencia, permanecen en la escuela un único curso porque pese a que consideran que en la escuela rural tienen mayor autonomía y flexibilidad organizativa, suelen estar en condiciones de interinidad y sueñan con trabajar con un único nivel educativo en el aula que responda a su ideal formativo de magisterio (Bustos, 2011; García, 2015). Sin duda, para la mayoría de docentes, la escuela rural es la primera experiencia docente (Sáinz, De León Elizondo y Santamaría, 2013).

En relación al alumnado de la escuela rural, apenas contamos con estudios específicos. Se sabe que en algunos centros rurales hay únicamente alumnado porque ha crecido en el pueblo, mientras en otras hay variabilidad, fruto del fenómeno de la inmigración y los trabajos temporeros de las familias (Gutiérrez, 2019). Dominan las ratios bajas y la individualización y se sabe que a los escolares les gusta la enseñanza multigrado porque prefieren interaccionar en el aula con otros de diferentes edades en lugar de con niños de la misma edad y además, la heterogeneidad aumenta el interés y la motivación, lo que se proyecta en una mayor participación activa en el aula (Abós y Boix, 2017; Grant, 2019) y en la adquisición de la capacidad para trabajar autónomamente (Boix y Bustos, 2014). También se sabe que a la mayoría del alumnado de la escuela rural andaluza, aragonesa y catalana le gustaría optar en un futuro a un trabajo perteneciente al sector servicios y que consideran que deben formarse más allá de la enseñanza obligatoria (Lorenzo, Domingo y Tomé, 2017).

Aunque, por lo general, las relaciones entre familias y escuelas son más frecuentes y estrechas en los centros rurales que en los urbanos, estas escuelas necesariamente deben estrechar lazos de colaboración, abrirse al contexto y conocer y valorar el entorno, las tradiciones, los valores y creencias específicas de la comunidad local sobre la educación (Álvarez y Vejo, 2017; Li, 2019; Vigo y Soriano, 2015). Para las familias la escuela rural en algunas regiones fue sinónimo de escuela de segunda, pero esta tendencia parece estar cambiando, aunque algunas familias siguen pensando que las escuelas urbanas tienen mayor "calidad" (Quílez y Vázquez, 2012; Raso, Marín 
y Rodríguez, 2017). No obstante, las familias de las escuelas rurales siguen teniendo una visión de la escuela muy ligada a lo asistencialista y con frecuencia realizan demandas al profesorado que superan sus competencias docentes (Alvarez-Alvarez y Ugarte-Higuera, 2019).

En base a todas estas ideas brevemente comentadas que nos ofrece la investigación científica sobre la escuela rural se centra este estudio, revisando su actualidad y vigencia hoy en dos provincias españolas.

\section{Método}

Se trata de una investigación compartida de una comunidad de docentes e investigadores basado en el compromiso, el intercambio y el apoyo mutuo para compartir significados y conocimientos de forma colaborativa. Esta investigación aborda ámbitos territoriales distantes y distintos (incluyendo escuelas rurales graduadas o multigrado). Por la idiosincrasia del estudio y con la finalidad de obtener datos generalizables y matizar las tendencias se consideró que lo más adecuado era proponer un estudio mixto (cuantitativo y cualitativo).

\section{Овjetivo}

El objetivo general es comparar la situación actual del entorno, los centros, el profesorado, el alumnado y las familias en escuelas rurales de diversa tipología en Cantabria y Huelva, para conocer sus semejanzas y diferencias.

\section{Participantes e instrumentos}

Dado el gran número de centros existente y su dispersión geográfica así como el ambicioso número de variables de interés se decidió que lo más conveniente era administrar un cuestionario (validez de contenido y de constructo, posteriormente se procedió a una validación de juicio de expertos) tipo Likert con cuatro alternativas posibles de respuesta: totalmente en desacuerdo, en desacuerdo, de acuerdo y totalmente de acuerdo adjudicando el valor asignado entre 1-4 a todos los centros rurales cántabros y onubenses. El instrumento administrado ha sido el empleado en el estudio de García (2015) debido a que ha seguido los procesos de validación oportunos -constructo, contenido, criterio y prueba piloto- siendo el alfa de Cronbach del cuestionario de 0,846, reflejando un alto índice de fiabilidad. Se compone de un total de 76 ítems agrupados en categorías para su tratamiento, de los cuales forman parte para este estudio 33. Además, se han incluido preguntas sobre los participantes, posibilitando un mayor número de correlaciones con las variables propias del cuestionario. Para que las personas que nos lo hiciesen llegar pudiesen aclarar o matizar cuanto estimasen conveniente, se incluye al final una pregunta abierta.

En el envío del cuestionario se ha realizado una selección de la muestra de posibles centros participantes, tratando de que se completase al menos un cuestionario por centro. Los criterios han sido: 
- Llegar al total de los Centros Rurales Agrupados (CRAs, Cantabria), Colegios Públicos Rurales (CPRs, Huelva) para tratar de lograr el máximo de respuestas de Centros de Educación Infantil y Primaria tanto en Cantabria como en Huelva. (En Cantabria hubo mortandad de muestra pese a que los cuestionarios fueron remitidos a los centros por correo electrónico en tres ocasiones y se efectuaron Ilamadas de teléfono a los centros que no respondieron animándoles a participar en el estudio. En Huelva, por el contrario, respondieron todos los centros).

- Centros ubicados en núcleos con población inferior a los 2000 habitantes. (En Cantabria, de los centros de una línea considerados rurales por la Consejería de Educación se ha tenido que desestimar uno por esta razón. En Huelva se obtuvo la totalidad de la población).

- Centros que impartan la etapa de Educación Primaria. (En Cantabria, de los centros de una línea considerados rurales por la Consejería de Educación se han tenido que desestimar ocho por impartir únicamente Educación Infantil. En Huelva, se ha acudido a la totalidad de escuelas rurales unitarias y, además, a los dos programas de infantil en casa que abarca diversas aldeas).

Tabla 1. Número de centros y cuestionarios en Cantabria

\begin{tabular}{|l|c|c|}
\hline \multicolumn{1}{|c|}{ Cantabria } & $\begin{array}{c}\text { Número de centros que } \\
\text { cumplen las condiciones }\end{array}$ & $\begin{array}{c}\text { Número cuestionarios } \\
\text { recogidos }\end{array}$ \\
\hline Centros Rurales Agrupados (CRA) & 4 & 4 \\
\hline Escuelas unitarias & 5 & 4 \\
\hline $\begin{array}{l}\text { Centros de Educación Infantil y Pri- } \\
\text { maria (CEIP) }\end{array}$ & 32 & 23 \\
\hline Total & $\mathbf{4 1}$ & $\mathbf{3 1}$ \\
\hline
\end{tabular}

Tabla 2. Número de centros y cuestionarios en Huelva

\begin{tabular}{|l|c|c|}
\hline \multicolumn{1}{|c|}{ Huelva } & $\begin{array}{c}\text { Número de centros que } \\
\text { cumplen las condiciones }\end{array}$ & $\begin{array}{c}\text { Número cuestionarios } \\
\text { recogidos }\end{array}$ \\
\hline Colegios Públicos Rurales (CPR) & 9 & 91 \\
\hline $\begin{array}{l}\text { Escuelas unitarias + Programa In- } \\
\text { fantil en Casa }\end{array}$ & 4 & $5+2$ \\
\hline $\begin{array}{l}\text { Centros de Educación Infantil y Pri- } \\
\text { maria (CEIP) }\end{array}$ & 18 & 156 \\
\hline Total & $\mathbf{3 1}$ & $\mathbf{2 5 4}$ \\
\hline
\end{tabular}

En Cantabria los centros han remitido un cuestionario, mientras en Huelva ha habido centros que lo han difundido entre el profesorado logrando más de una respuesta por escuela. 
Siguiendo el principio de triangulación metodológica y para saturar informaciones, también hemos procedido a realizar entrevistas en profundidad con informantes clave, ajustadas a las categorías establecidas. Para seleccionar a los participantes, los criterios han sido:

- Contactar con directores y maestros rurales (tutores e itinerantes) de Centros Rurales Agrupados (CRAs, Cantabria) y Colegios Públicos Rurales (CPRs, Huelva).

- Contactar con profesorado de Centros graduados de Educación Infantil y Primaria tanto en ambas provincias.

- Contactar con profesorado de aulas unitarias en ambas provincias.

- Contactar con el director del CRIE de Cantabria.

- Seleccionar centros situados en localidades próximas a la costa, en los valles y en la alta montaña.

En cuanto a las entrevistas, los participantes se distribuyen del siguiente modo:

Tabla 3. Participantes en entrevistas en Cantabria y Huelva

\begin{tabular}{|l|l|}
\hline \multicolumn{1}{|c|}{ Provincia } & \multicolumn{1}{c|}{ Participantes } \\
\hline Cantabria & 2 directoras de CRA \\
& 3 maestras rurales de CRA \\
& 1 maestra itinerante de CRA \\
& 2 directoras de CEIP rural (1 en aula unitaria) \\
& 2 maestras de CEIP rural \\
& 1 director de CRIE \\
\hline Huelva & 9 directores-as de CRA -CPR- \\
& 5 maestros-as de CRA \\
& 4 directoras de escuelas unitarias \\
& 18 directores-as de CEIP rural \\
& 5 maestros-as de CEIP rural \\
\hline Total & $\mathbf{1 1}$ informantes cántabros \\
& $\mathbf{4 1}$ informantes onubenses \\
\hline
\end{tabular}

Las preguntas realizadas en las entrevistas estaban íntimamente relacionadas con las variables del cuestionario enumeradas anteriormente, profundizando en las posibilidades y límites de cada realidad escolar.

\section{ANÁlISIS Y TRATAMIENTO DE DATOS}

Tras la recogida de información se organizaron y analizaron los datos empleando el programa SPSS v.21 para los datos cuantitativos. En un primer momento, se realizaron análisis descriptivos básicos de porcentajes, frecuencias y medidas de tendencia central de las variables observadas para este estudio. 
El programa Atlas-ti v.6.2 se utilizó para los datos cualitativos, mediante la configuración de una tabla hermenéutica con las categorías establecidas previamente en el estudio -documentos primarios, citas, códigos, etc.-- Una vez analizada la información, para su interpretación, fueron codificadas las citas textuales de los participantes (en el ejemplo: 3:11, 78:81, D3, tenemos: 3:11 se refiere al número de entrevista y el número de cita dentro de ese documento, 78:81 indica el párrafo donde se inicia y termina la cita y, por último, D3, señala al participante (Director).

\section{Resultados}

Para presentar los resultados los hemos organizado en torno a cinco grandes categorías: 1) entorno, 2) centros, 3) profesorado, 4) alumnado y 5) familias. Al tratarse de una investigación compartida de ámbitos territoriales distintos y distantes, se mostrarán gráficos comparativos respecto a las prácticas y concepciones estudiadas. Previamente, se ofrece una contextualización sociodemográfica de los participantes.

La escuela rural de Cantabria y de Huelva vista con visión panorámica mantiene el isomorfismo, ya que la tendencia es muy similar, pero la comparativa de variables, así como sus correlaciones, permite extraer alguna información diferenciada significativa.

\section{CONTEXTUALIZACIÓN SOCIODEMOGRÁFICA}

Se muestran dos territorios distintos con propensión semejante. En este panorama compartido que se dibuja, se puede observar que las plantillas docentes en Cantabria tienen más estabilidad, mayor experiencia docente en centros rurales (en Huelva la tendencia en estos centros es de tránsito en la mayoría de los casos), excepto el profesorado que reside en el pueblo articulando su proyecto vital. Es recurrente incluirlo entre los obstáculos que citan los docentes: "un obstáculo es que la plantilla no es definitiva, no hay continuidad en el profesorado" (2:3, 12:13, D2).

Igualmente, en Cantabria, indican que Ilevan ejerciendo más tiempo en su centro actual, así como es relevante el dato de preferencia de ejercer en centros rurales ( $87 \%$ en Cantabria frente al $41,9 \%$ en Huelva). Este último dato tiene que ver con las distancias y la dispersión de los pueblos en Andalucía.

Entre otros datos cabe destacar un contraste evidente: en Cantabria la media de edad del profesorado es más elevada, hay una mayor estabilidad en la zona rural, un $83,9 \%$ del profesorado están en situación administrativa definitiva, prefieren ejercer en centros rurales, etc. mientras en Huelva los datos son menos alentadores. Esta diferencia está relacionada con que en Cantabria los pueblos están más cerca unos de otros y hay centros comarcarles: aunque ha bajado la población rural de los pueblos pequeños, sin embargo, los de cabecera de comarca han subido. En Andalucía, dada la extensión del territorio, las mismas variables se comportan de manera distinta. 
Tabla 4. Contextualización de los participantes

\begin{tabular}{|c|c|c|c|}
\hline \multicolumn{2}{|c|}{ DATOS SOCIO-DEMOGRÁFICOS } & HUELVA & CANTABRIA \\
\hline \multicolumn{2}{|l|}{ Edad (Media) } & 39,33 & 45,71 \\
\hline \multirow{2}{*}{ Sexo $(\%)$} & Hombre & $31 \%$ & $25,8 \%$ \\
\hline & Mujer & $69 \%$ & $74,2 \%$ \\
\hline \multicolumn{2}{|l|}{ Años de experiencia docente (Media) } & 11,81 & 18 \\
\hline \multicolumn{2}{|l|}{ Tiempo ejerciendo en el centro actual (Media) } & 5,63 & 10,29 \\
\hline \multirow{3}{*}{ Situación administrativa (\%) } & Interino & $36,4 \%$ & $9,7 \%$ \\
\hline & Provisional & $13,6 \%$ & $6,5 \%$ \\
\hline & Definitivo & $50 \%$ & $83,9 \%$ \\
\hline \multirow{2}{*}{$\begin{array}{l}\text { Reside en el pueblo en el que imparte do- } \\
\text { cencia }(\%)\end{array}$} & Sí & $28,7 \%$ & $12,9 \%$ \\
\hline & No & $71,3 \%$ & $87,1 \%$ \\
\hline \multirow{3}{*}{ Preferencia de ejercer en $(\%)$ : } & Escuelas rurales & $41,9 \%$ & $87,1 \%$ \\
\hline & Escuelas urbanas & $9,3 \%$ & $0 \%$ \\
\hline & Es indiferente & $48,8 \%$ & $12,9 \%$ \\
\hline \multirow{3}{*}{$\begin{array}{l}\text { ¿Considera que su centro tiene característi- } \\
\text { cas rurales? }(\%)\end{array}$} & Sí & $94,2 \%$ & $93,5 \%$ \\
\hline & No & $5,8 \%$ & $3,2 \%$ \\
\hline & Mixto & $0 \%$ & $3,2 \%$ \\
\hline \multirow{3}{*}{ Me considero (\%): } & $\begin{array}{l}\text { Maestro } \\
\text { tradicional }\end{array}$ & $5,8 \%$ & $6,5 \%$ \\
\hline & $\begin{array}{l}\text { Maestro } \\
\text { Innovador }\end{array}$ & $22,5 \%$ & $29 \%$ \\
\hline & $\begin{array}{l}\text { Entre los dos ante- } \\
\text { riores }\end{array}$ & $71,7 \%$ & $64,5 \%$ \\
\hline \multirow{4}{*}{ Lugar de nacimiento (\%) } & Aldea & $2,3 \%$ & $6,5 \%$ \\
\hline & Pueblo pequeño & $36,8 \%$ & $41,9 \%$ \\
\hline & Pueblo grande & $21,7 \%$ & $25,8 \%$ \\
\hline & Ciudad & $39,1 \%$ & $25,8 \%$ \\
\hline \multicolumn{2}{|l|}{ Número de alumnos por clase (Media) } & 11,27 & 12,45 \\
\hline
\end{tabular}

También destaca la auto consideración de los docentes como "tradicionales-innovadores", situándose las respuestas en un planteamiento "deseado". Tras realizar diversas correlaciones se pueden encontrar determinadas incoherencias como, por ejemplo: maestro que resalta que es innovador y utiliza el libro de texto como eje central de la clase. Igualmente, se puede observar como alrededor del $40 \%$ del profesorado de la escuela rural pese a que ha nacido en aldeas o pequeñas localidades quieren terminar en poblaciones mayores (capitales comarcales, capitales de provincia, pueblos con más de 10000 habitantes). Asimismo, profesorado nacido en poblaciones mayores quiere disfrutar del medio y la escuela rural como experiencia profesional, y en muchos casos, personal. 
En el análisis de la escuela rural en Cantabria y Huelva, como se puede observar a continuación, la tendencia es parecida, pero presenta diferencias que las singularizan, pero también se observan divergencias.

\section{ENTORNO}

La pérdida de población en los pequeños pueblos está causando la disminución de servicios básicos para el mantenimiento de las personas -médicos, bancarios, farmacéuticos, correos, etc.-, viéndose acuciada por la crisis del sector agrícola y ganadero.

Como se puede observar (ver figura 1), las mayores desviaciones se encuentran en el ítem 3 (22,6 \% de acuerdo frente al 51,9\% en Huelva) y el ítem 4 (9,7\% profesorado cántabro en niveles de acuerdo debido a la cercanía entre pueblos mientras que en Huelva se incrementa hasta el 22,9 de los participantes) debido a que en Cantabria no existe subsidio agrícola -Extremadura y Andalucía son las únicas Comunidades Autónomas-. En la percepción docente, es una prioridad cambiar hacia políticas sociales sostenibles, basadas en el territorio y no tanto en el sujeto (por ejemplo, el paro agrícola -PER-).

Aunque existe mayor distancia entre localidades rurales de Huelva por su extensión geográfica, los centros rurales de Cantabria se enclavan en una orografía montañosa con vías de comunicación precarias. En las dos provincias señalan que el entorno sociocultural de la escuela es un medio más conservador que el urbano (ítem 1.c), que ofrece posibilidades por su idiosincrasia: "su disposición espacial y atención al alumnado, relaciones con la familia" (14:6, 23:24, D14).

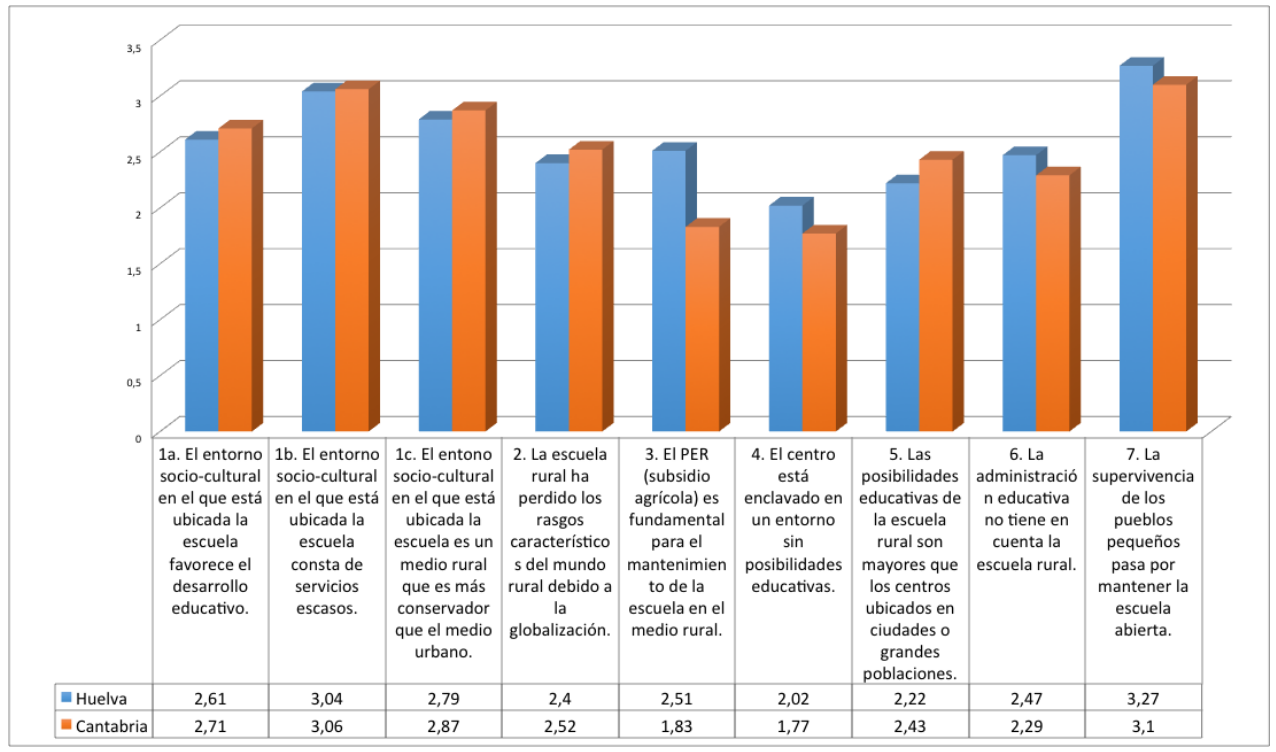

Figura 1. Resultados de la categoría entorno 
Entre centros de la misma provincia en las entrevistas se muestran diferentes contrastes. En Cantabria: "Se trata de un espacio rural en transición. Cambio de sectores económicos, del primero al tercero" (7:2, 8:10, D7). "Es un entorno rural tradicional. Aquí no hay nada. Solo hay dos bares, no hay más. Un día a la semana sube un pescadero y otro día sube una frutera" (1:3, 5:7, D1). "Estamos en un contexto rural moderno. Porque aun siendo un pueblo pequeño, la mayor parte de la población no es ganadera, hay muchas personas que se dedican al sector servicios como el comercio" $(8: 7,12: 13$, D8). En Huelva encontramos mayor número de escuelas que se consideran insertas en medios tradicionales o en transición. Es evidente que los entornos rurales y sus centros educativos, están transformándose: "el entorno del centro mantiene características de la escuela rural, pero está en continuo cambio, aunque hay actividades agrarias, también existen otra de la población que se dedica al sector servicios" (15:8, 11:12, D15).

\section{Centros}

A nivel de centros se observa que no hay rupturas en la propensión. Las diferencias, por tanto, no son significativas, aunque en Huelva se apunta a una implicación menor en actividades externas al colegio, correlacionando el dato con los participantes que viven en el mismo pueblo en el que trabajan (12,9\%) así como la situación administrativa (definitivos el 83,9 \%), que es mayor en Cantabria, de ahí, una mayor participación. En Huelva, la plantilla es más inestable $(50 \%$ son definitivos).

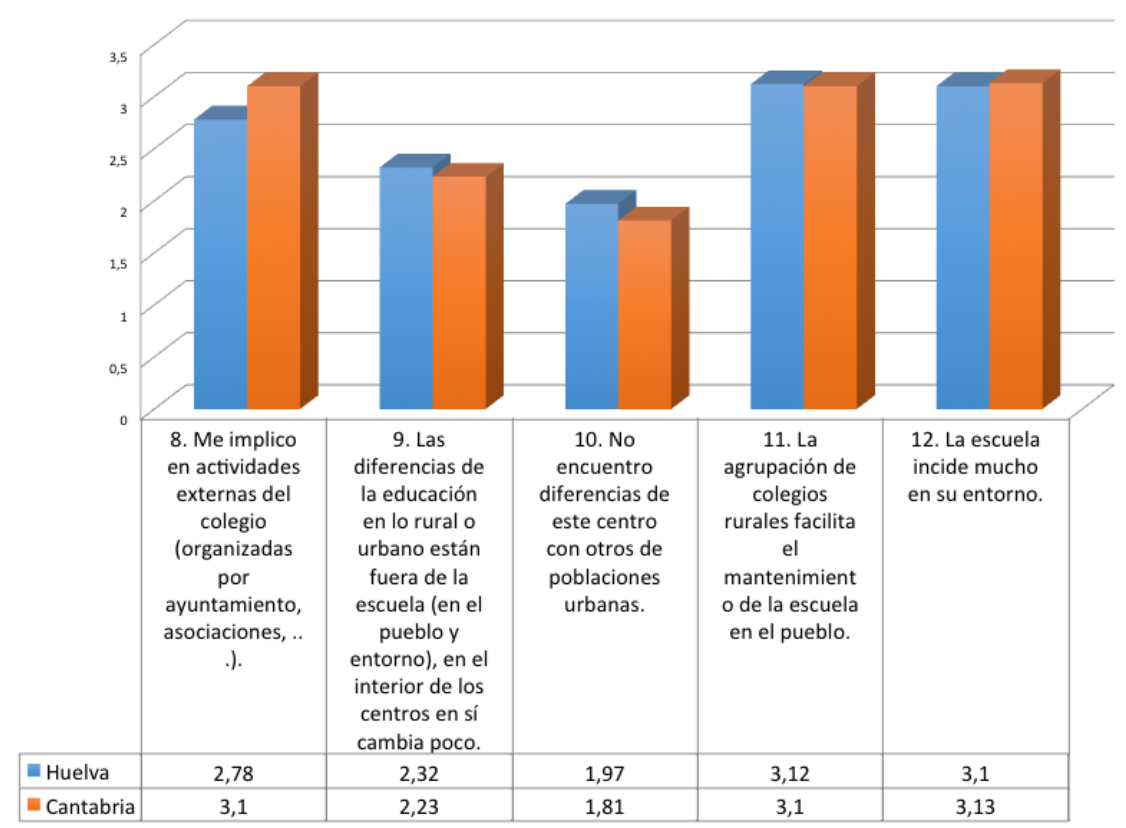

Figura 2. Resultados de la categoría Centros I 
La supervivencia de la escuela rural ayuda a enraizar a la población porque dinamiza culturalmente al pueblo e incide al profesorado en la movilización cultural (ítem 10). Perciben como positivo cuando las escuelas rurales se agrupan (ítem 11) y subrayan el valor del territorio y de la comarca.

La supervivencia de la escuela rural es necesaria para arraigar a la población porque dinamiza culturalmente y porque el profesorado incide a través de su acción educativa en el medio y se desarrolla de manera más efectiva cuando los centros, en vez de permanecer anclados en su localidad, son capaces de conectarse.

También es importante reseñar la demanda de recursos en su comparativa con la escuela urbana (ítem 14), aspecto que también fue destacado en las entrevistas: "es un centro muy alejado de cualquier localidad urbana y escasez de recursos" (3:8, 11:12, D3).

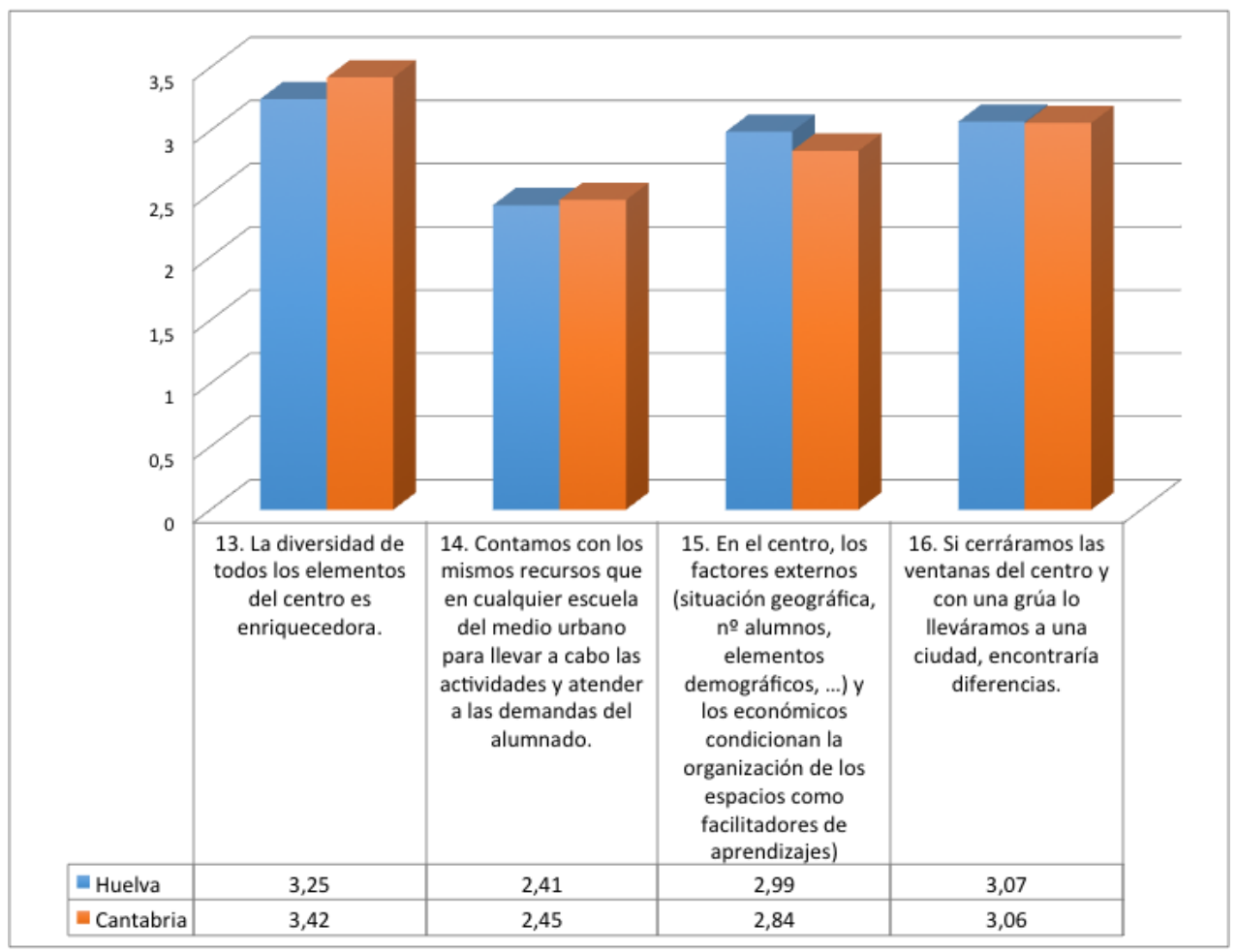

Figura 3. Resultados de la categoría Centros II

En definitiva, la escuela rural es diversa y sigue siendo diferente a la urbana, pero sus rasgos parece que se van perdiendo en favor de una mayor homogeneidad.

\section{Profesorado}

Los docentes de las dos provincias manifiestan una misma tendencia, pero con matices (ver figura 4). El profesorado es relativamente optimista y se autodefine como 
implicado, y dicen haber elegido el medio rural para su desarrollo (ítem 18). En este sentido, distan Cantabria y Huelva, ya que en la primera se pronuncian en este sentido prácticamente el $100 \%$ de los participantes (71\% en los niveles de totalmente de acuerdo y el 25,8\% de acuerdo).

El profesorado se siente reconocido por la población a la que presta sus servicios (ítem 19), con satisfacción profesional (ítem 20) y en contacto cercano con las familias. No se siente cohibido, que es uno de los tópicos de los maestros en el pueblo, aunque un 25-30\% muestran rechazo por la pérdida de anonimato (ítem 21). De alguna manera, todas las personas se conocen, pero esto también puede suceder en los barrios de las ciudades.

Otra de las cuestiones que no sigue la tendencia en este estudio comparativo, es la de la escuela rural como centros educativos de tránsito (ítem 17.a). Mientras en Cantabria afecta a un $13 \%$ de docentes, en Huelva se incrementa significativamente hasta un 60 \%. Así se pone de manifiesto en las entrevistas: "la mayor parte del profesorado es interino o provisional, y está solo un año en el colegio" (12:23, 56:57, ED12),

En general, se encuentran satisfechos, pero creen que se podría mejorar respecto a la formación universitaria: "La formación adquirida en la universidad no te prepara para trabajar en contextos rurales" (5:11, 23:25, ED5). La mayoría del profesorado de las dos provincias se desvincula del axioma de formación específica de los docentes. Al respecto, se indica que: "no podemos formar para cada caso concreto en la universidad, aunque sí proponer asignatura, cursos específicos y prácticas en los pueblos" (16:18, 45:46, ED16); "Únicamente formar, luego son las ganas de trabajar. Es en la práctica donde se aprende y se aprenden estrategias metodológicas" (2:9, 32:33, D2); "Profesionales implicados, activos y participativos" (7:14, 28:29, D7).

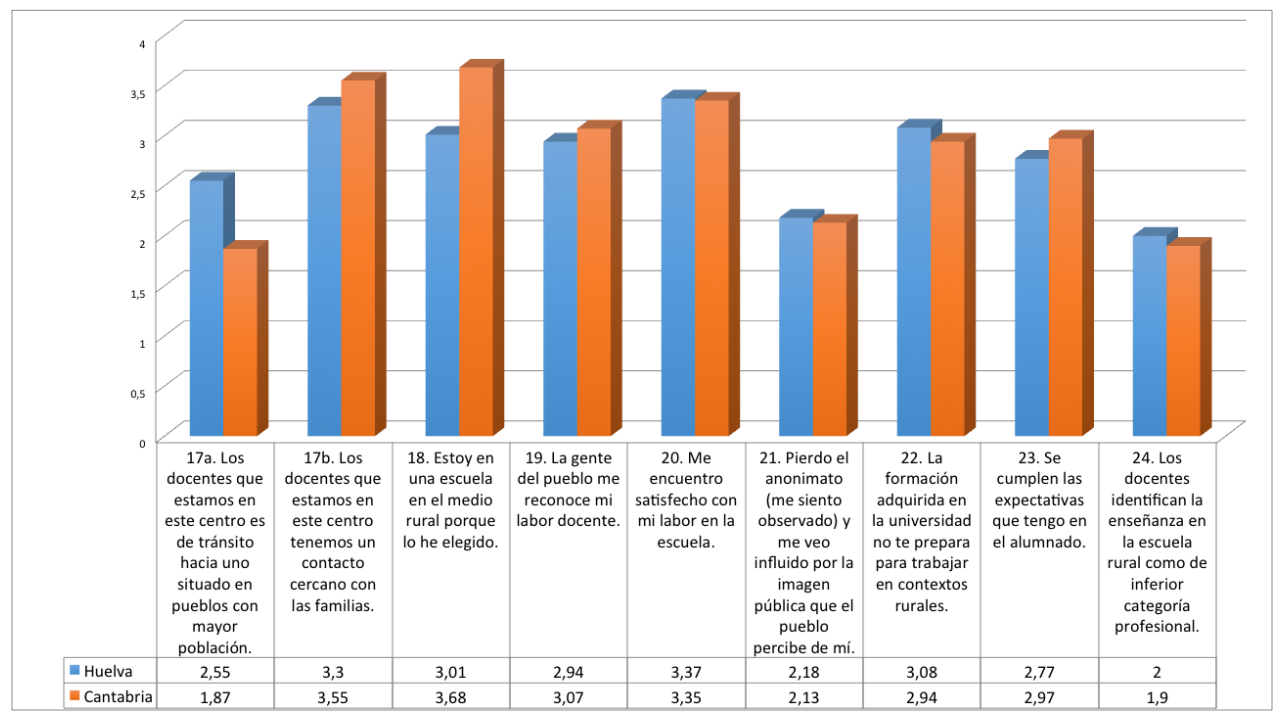

Figura 4. Resultados de la categoría profesorado 


\section{Alumnado}

Las mayores diferencias que se encuentran en la presente categoría en sentido comparativo (ver figura 5), aluden a la formación (ítem 25.b). Uno de los problemas tradicionales que se pone de relieve es la transición de la escuela rural a los Institutos de Educación Secundaria situados en la cabecera comarcal. Los participantes han comentado: "el principal problema que veo aquí es que los alumnos en Secundaria se tienen que ir internos a otra localidad, de lunes a viernes. No hay alternativa. El pueblo está a 50 minutos por una carretera que es curva contra curva. Se tienen que ir" $(2: 45,56: 57, E D 2)$. También varían los intereses respecto a las temáticas del libro de texto (ítem 26). No obstante, las variables más significativas con altos índices de acuerdo, son las que se refieren a la respuesta educativa adaptada al alumnado que se facilita en la escuela rural (ítem 25.a) y la consideración nuevamente de la preparación para afrontar estudios de secundaria (formación).

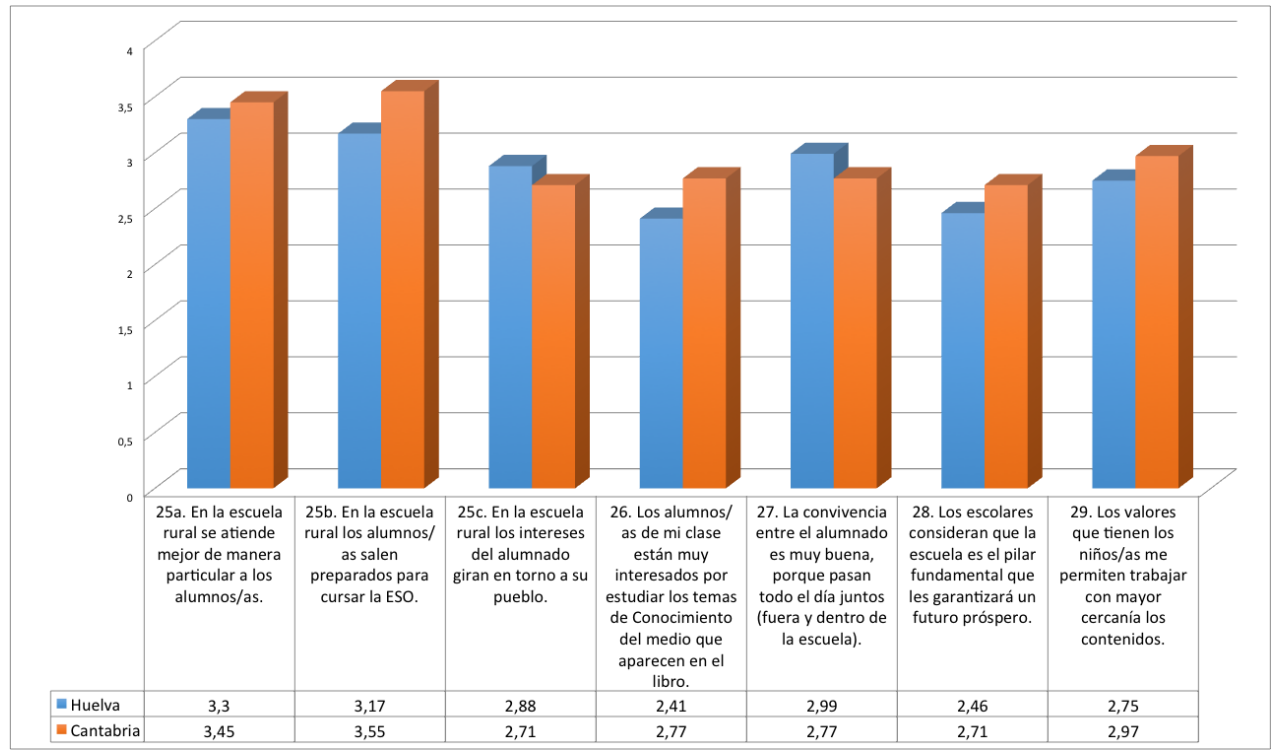

Figura 5. Resultados de la categoría alumnado

En líneas generales, los docentes consideran que el alumnado está bien preparado y que puede seguir sus estudios sin problemas ninguno. Identifican que los escolares tienen intereses demasiado localistas, lo que nos habla de la importancia de la escuela rural que tiene un reto ante la globalización, sin perder la identidad local, es decir, combinar lo local y lo global. También destacan la buena convivencia que suele darse en los centros, con lo cual nos dice que es un alumnado que está suficientemente preparado y que convive bien.

Algunas evidencias respecto a alumnado y la cercanía de los mismos: "Son muy autónomos, independientes, cooperativos (los mayores ayudan a los pequeños), proponen, son creativos..." (6:16, 62:63, D6); "En el patio están todos juntos. Por lo 
general, no hay incidencias de conflictos, ni de atención ni de convivencia" (11:34, 24:25, D11); "Les definiría como una gran familia en la que todos se ayudan y se cuidan" (6:31, 27:28, D6); "La convivencia de diferentes edades en una misma aula hace que se creen lazos sociales muy especiales. La socialización de todo el alumnado es muy positiva" (10:15, 16:17, D6).

\section{FAMILIAS}

Si hacemos una lectura de todos los datos de esta categoría en su conjunto, (ver figura 6), los más significativos son los ítems 30.a, referente a la participación y el 31 , sobre las relaciones con las instituciones locales. A nivel comparativo entre los dos casos, las mayores desviaciones se dan en el ítem 30.d, sobre las expectativas. Los participantes destacan de la familia un tono bajo de participación, si bien no se trata de un problema específico del medio rural, sino de la sociedad contemporánea. Los docentes señalan que las familias participan tanto en la escuela como en el aula, habiendo margen para seguir avanzando. También es relevante el ítem 32, que transmite un cambio laboral, social y cultural del tradicional maestro de escuela rural a un perfil más funcionarial que finaliza el compromiso con el contexto cuando ha terminado la jornada. En el ítem 33, se percibe un contraste entre el profesorado rural por parte de las familias, otorgando un carácter asistencialista mayor en la provincia de Huelva con datos del 54,7 \% frente al 25,8 \% de docentes cántabros.

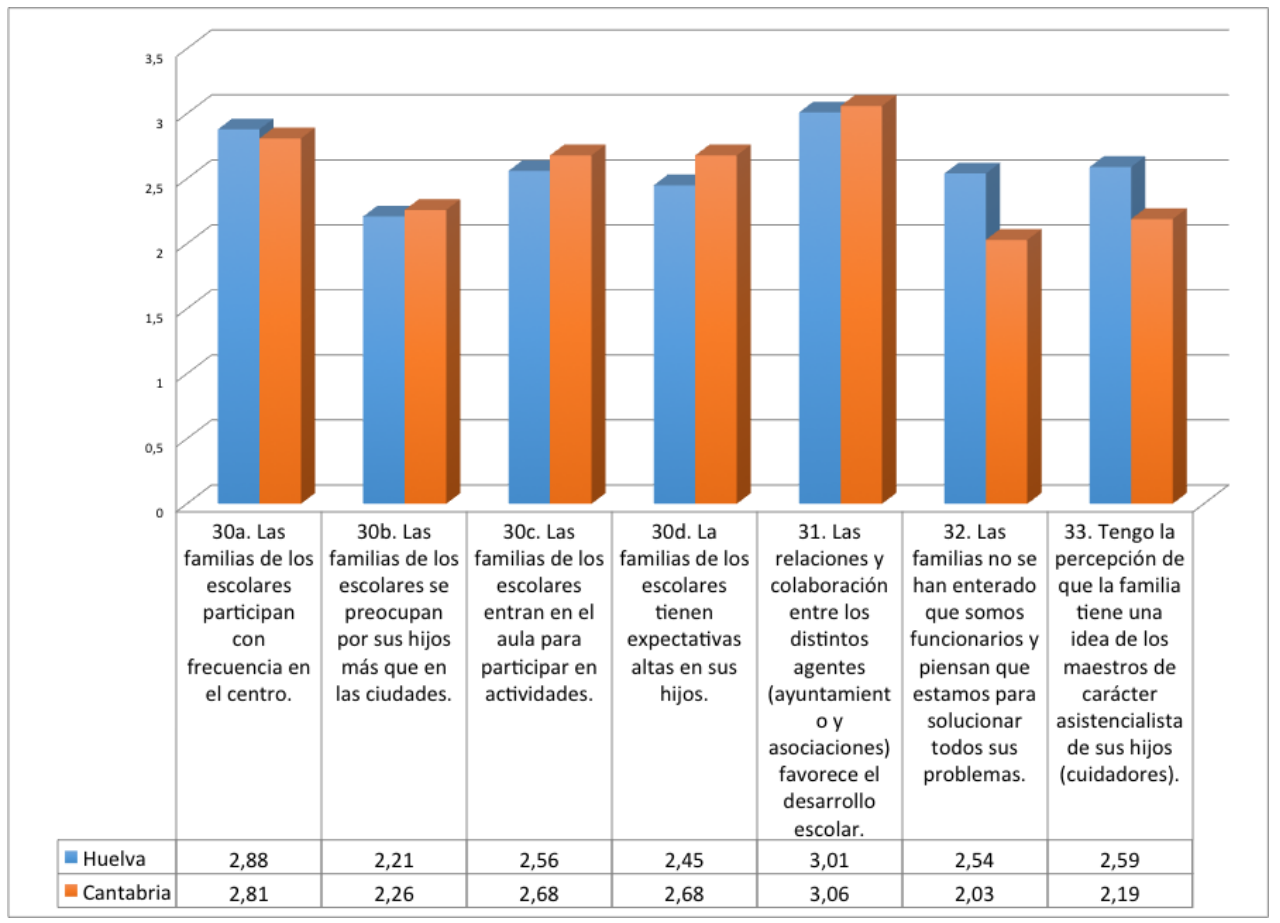

Figura 6. Resultados de la categoría familias 
En torno a la comunidad rural se suele evaluar el nivel de formación, de participación, expectativas, etc. de las familias. Así se manifestaron los participantes al respecto: "El nivel cultural es bajo. Hay de todo. Están implicadas, pero hay familias que tienen muy altas expectativas de sus hijos y las hay que se conforman con que sepan leer y escribir" (1:29, 62:63, D1); "Suelen colaborar en actividades varias que organiza el centro (convivencia, talleres...)" (12:24, 86:87, D12); "Hay mucho contacto directo con las familias y eso hace que se impliquen más" (17:42, 65:66, D17).

\section{Conclusiones}

Desde una perspectiva panorámica las tendencias de los resultados nos muestran dos territorios distintos con propensión semejante en la mayoría de las variables estudiadas (entorno, centros, profesorado, alumnado y familias), verificándose nuestra hipótesis de partida: que habría semejanzas, pero también diferencias entre el norte (Cantabria) y el sur (Huelva) de España.

Respecto al entorno en el que se encuentran las escuelas rurales en Cantabria los pueblos están más cerca unos de otros y aunque ha bajado la población rural de los pueblos pequeños, sin embargo, los de cabecera de comarca han subido, existiendo centros comarcarles. Destaca la pérdida paulatina de servicios y se observa que el arraigo o sentimiento de pertenencia ya no es suficiente, por lo que parece necesario encontrar respuestas para cubrir las necesidades primarias y mantener la población en los pueblos (Benito, 2013; Bustos, 2011; VázquezRecio, 2016). En este sentido, los territorios necesitan de la escuela rural para arraigar su población y contar con una política comarcal comunitaria que apoye el valor de las comarcas y sus singularidades. Para que esta escuela rural impacte en el territorio es positivo agruparla, para que cuente con más medios, recursos y posibilidades. Asimismo, tiene sentido reivindicar las mancomunidades, ya que pueden ayudar a superar el localismo y generar mayores conexiones y desarrollo, abordando estrategias territoriales para el desarrollo comarcal, y esta es una de las claves, abordar la estrategia territorial desde el desarrollo de la comarca. Se están desarrollando semejantes políticas rurales y medidas económicas, con pequeñas diferencias como es el PER.

Respecto a los centros, observamos que la escuela rural es diversa y tiene una riqueza que hace que todas sus características incidan en la realidad local (Martínez y Bustos, 2011). Se observa una implicación mayor en las actividades externas al colegio en Cantabria. Sin embargo, comparten un reto: ser capaces de ofrecer un servicio de calidad para la comunidad a la que sirven, adaptándose a las características y peculiaridades de las personas y del lugar donde se enclavan. Asimismo, la agrupación de escuelas rurales sigue siendo una política necesaria y es necesario que la Administraciones públicas sigan creyendo en el modelo educativo rural.

Respecto al profesorado, es optimista, está en el entorno rural porque quiere aunque en menor medida en Huelva-, suficientemente reconocido y satisfecho, en contacto con la comunidad, y formación mejorable. No obstante, la escuela rural en Andalucía está copada por interinos, mientras que en Cantabria son plantillas 
más estables, contradiciendo en parte los resultados de Sánchez, Martínez y Lucena (2017), ya que en Cantabria parece haber una mayor preferencia por los centros rurales, aunque se debería investigar más a fondo. En línea con esto, nuestros resultados coinciden con los del estudio de Pérez, García y Quijano (2018) sobre la realidad actual de los colegios rurales de Andalucía pues se observa cierto desarraigo con la comunidad cultural. Asimismo, valoran el valor del territorio y de la comarca, lo que coincide con diversos estudios (Heredero, Collado-González y Shoiti-Nozu, 2016; Raso, Marín y Rodríguez, 2017; Tahull y Montero, 2018). En la mayoría de los casos valoran como gratificante la relación y bienestar con la comunidad educativa, coincidiendo con estudios previos (Campos, 2016; Sánchez, Martínez y Lucena, 2017). La mayoría del profesorado de los dos casos objeto de estudio se desvincula del axioma de formación específica de los docentes (Campos, 2016; Magro, 2019), lo cual puede ser positivo, ya que se puede correr el riesgo de formar para un entorno específico creando determinismos (Chaparro y Pastor, 2018).

Respecto al alumnado, se observa que está bien dispuesto para el aprendizaje, sale de la escuela preparado para la Secundaria, mantiene buenas relaciones de convivencia y una identidad localista, pero con una visión global, algo que se ha demostrado en los distintos estudios que dan la voz al alumnado rural (Núñez, Peña, Cubillos y Solorza, 2016).

Respecto a las familias se observa que tienen una participación suficiente, pero los maestros desearían una mayor implicación cultural, siendo portadora de conocimiento a la escuela. También se observa que la población no se corresponde necesariamente con perfiles conservadores desde el punto de vista social, asemejándose a la que se puede encontrar en otros entornos (Abós, 2015). No son conformistas, ya que se demanda algo más que una formación elemental para sus hijos que les abra oportunidades y que les iguale con otras poblaciones (Tahull y Montero, 2018) poniendo en cuestión que la calidad de los centros urbanos sea mayor (Quílez y Vázquez, 2012; Raso, Marín y Rodríguez, 2017). La comunidad educativa de la escuela rural, se caracteriza por identificarse a sí misma como diferente a la urbana, pero está perdiendo paulatinamente esos rasgos hacia una mayor homogeneidad. Asimismo, la implicación de las familias debería ir más allá del acompañamiento para ganar una implicación sociocultural mayor (Alvarez-Alvarez y Ugarte-Higuera, 2019).

En conclusión, podríamos decir que ni la escuela rural ni los entornos rurales atraviesan su mejor momento ni en el norte ni en el sur de España. Se observan mayores contrastes en el entorno y el profesorado, pero similitudes en los centros, el alumnado y las familias entre los diferentes tipos de centros educativos rurales Huelva y Cantabria. Las escuelas están menos aisladas y cuentan con un profesorado más estable en Cantabria. En ambas provincias el profesorado se muestra optimista y cuentan con un alumnado bien dispuesto. La participación de las familias es mejorable, pero tienen altas expectativas en la escuela, la cual consideran que debe adaptarse a los cambios de la sociedad. El reto es y sigue siendo asegurar un sistema educativo equitativo que ayude a mantener vivo el mundo rural ofreciendo una educación de calidad a todos los estudiantes, con independencia de su procedencia (Gallardo, 2011). 


\section{REFERENCIAS BIBLIOGRÁFICAS}

Abós, P. (2015). El modelo de escuela rural ¿es un modelo transferible a otro tipo de escuela? Educação \& Realidade, 40(3), 667-684. Recuperado de http://www. scielo.br/scielo.php?script=sci_arttext\&pid=S2175-62362015000300667.

Abós, P. y Boix, R. (2017). Evaluación de los aprendizajes en escuelas rurales multigrado. Aula Abierta, 45, 41-48. http://doi.org/10.17811/rifie.45.2017.41-48. Abós, P., Boix, R., Bustos, A., Domingo, P. L., Domingo, C. V. y Ramo, R. M. (2015). La escuela rural y la política educativa española. Diferencias entre comunidades autónomas. International Studies on Law and Education, 19, 73-90. Recuperado de https://zaguan.unizar.es/record/56204?ln=es.

Álvarez-Álvarez, C. y Ugarte-Higuera, A. (2019). La escuela graduada en el medio rural. Un estudio de caso. Revista de Educación de la Universidad de Granada, 26, 163-181. http://doi.org/10.30827/reugra.v26i0.154.

Álvarez, C. y Vejo, R. (2017). ¿Cómo se sitúan las escuelas españolas del medio rural ante la innovación? Un estudio exploratorio mediante entrevistas. Aula Abierta, 45, 25-32. http://doi.org/10.17811/rifie.45.2017.25-32.

Barthes, A., Champollion, P. y Alpe, Y. (2018). Evolutions of the Complex Relationship Between Education and Territories. Plymouth: ISTE editions.

Benito, D. (2013). Despoblación, desarraigo y escuela rural: condenados a encontrarse. Encrucijadas. Revista crítica de Ciencias Sociales, 6, 56-69. Recuperado de http://www.encrucijadas.org/index.php/ojs/article/view/49.

Boix, R. y Bustos, A. (2014). La enseñanza en las aulas multigrado: Una aproximación a las actividades escolares y los recursos didácticos desde la perspectiva del profesorado. Revista Iberoamericana de Evaluación Educativa, 7(3), 29-43. Recuperado de https://repositorio.uam.es/handle/10486/662851.

Burrial, X., Sala, T., Samper, L. y Torres, T. (2018). La educación en la sociedad rural. Una evaluación cualitativa de la escuela rural catalana. Intercambio/Échange, 2, 145-158. Recuperado de https://repositori.udl.cat/handle/10459.1/65427.

Bustos, A. (2011). La escuela rural. Granada: Octaedro.

Campos, J. (2016). Conocimiento profesional de los profesores: formación de profesores en dos agrupamientos de escuelas. Revista Electrónica Interuniversitaria De Formación Del Profesorado, 19(2), 273-287. https://doi.org/10.6018/ reifop.19.2.255681.

Chaparro, F. y Pastor, M. L. (2018). La formación del profesorado para la Escuela Rural: una mirada desde la educación física. Revista Electrónica Interuniversitaria de Formación del Profesorado, 21(3), 93-107. Recuperado de https://revistas.um.es/ reifop/article/view/321331.

Gallardo, M. (2011). La escuela de contexto rural: ¿de la diferencia a la desigualdad? Revista Iberoamericana de Educación, 55(5), 1-10. 
García, F. J. (2015). Escuela, medio rural y diversidad cultural en un contexto global: currículum, materiales didácticos y práctica docente de Conocimiento del Medio: situación, límites y posibilidades en centros onubenses. Recuperado de http://rabida.uhu.es/dspace/handle/10272/11440.

García-Valcárcel, A., Basilotta, V. y Mulas, I. (2016). Fomentando la ciudadanía digital mediante un proyecto de aprendizaje colaborativo entre escuelas rurales y urbanas para aprender inglés. Profesorado, 20(3), 549-581. Recuperado de https:// recyt.fecyt.es/index.php/profesorado/article/view/54613.

Grant, P. (2019). Book review of "College aspirations and access in working-class rural communities: The mixed signals, challenges, and new language first-generation students encounter." Journal of Research in Rural Education, 35(4), 1-4. https://doi. org/10.26209/jrre3504.

Gutiérrez, M. (2019). Necesidades formativas del profesorado de educación infantil para la atención a la diversidad de colegios rurales agrupados. Revista Prisma Social, 25, 103-125. Recuperado de https://revistaprismasocial.es/article/view/2683.

Hamodi, C. y Aragués-Garde, S. (2014). La escuela rural: ventajas, inconvenientes y reflexiones sobre sus falsos mitos. Palobra, 14, 46-61. Recuperado de http://ojs. udc.edu.co/index.php/palobra/article/view/631.

Heredero, E., Collado-González, I. y Shoiti-Nozu, W. (2016). Los colegios rurales agrupados en España. Analisis del funcionamiento y organización de la escuela rural española a partir de un estudio de casos. Educação e Fronteiras On-Line, 4(12), 142-153. http://doi.org/10.30612/eduf.v4i12.4716.

Li, X. (2019). Challenging both rural advantage and disadvantage narratives: The effects of family factors on American student college expectations in the early 2010s. Journal of Research in Rural Education, 35(5), 1-16. https://doi. org/10.26209/jrre3505.

Lorenzo, J., Domingo, V. y Tomé, M. (2017). Expectativas y creencias del alumnado rural sobre su futuro profesional y académico. Aula Abierta, 45, 49-54. http://doi. org/10.17811/rifie.45.2017.49-54.

Magro, M. (2019). Necesidades formativas del profesorado de Educación Infantil para la atención a la diversidad de Colegios Rurales Agrupados. Revista Prisma Social, 25, 103-125. Recuperado de https://revistaprismasocial.es/article/ view/2683.

Martínez, J. B. y Bustos, A. (2011). Globalización, nuevas ruralidades y escuelas. Profesorado, 15(2), 3-12. Recuperado de https://recyt.fecyt.es/index.php/profesorado/ article/view/43399.

Ministerio de Educación, Cultura y Deporte-Consejo Escolar del Estado (2015). Informe 2015 sobre el estado del sistema educativo (curso 2013-2014). Madrid: Secretaría General Técnica. Recuperado de http://www.mecd.gob.es/cee/publicaciones/informes-del-sistema-educativo/informe-2015.html. 
Núñez, C. G., Peña, M., Cubillos, F. y Solorza, H. (2016). Estamos todos juntos: el cierre de la Escuela Rural desde la perspectiva de los niños. Educação e Pesquisa, 42(4), 953-967. http://doi.org/10.1590/s1517-9702201612152334.

Pérez, M., García, I. y Quijano, R. (2018). Una aproximación a la realidad de los centros públicos rurales en Andalucía. Tendencias Pedagógicas, 32, 147-160. http:// doi.org/10.15366/tp2018.32.011.

Quílez, M. y Vázquez, R. (2012). Aulas multigrado o el mito de la mala calidad de la enseñanza en la escuela rural. Revista Iberoamericana de Educación, 59(2), 1-14. Recuperado de https://rieoei.org/RIE/article/view/1393.

Raso, F., Marín, J. A. y Rodríguez, A. M. (2017). Análisis de la satisfacción del profesorado de la escuela rural en la provincia de Granada (España) respecto a su relación personal y profesional con la comunidad educativa. European Scientific Journal, 13(4), 27-50. http://doi.org/10.19044/esj.2017.v13n4p27.

Sáinz, E., De León Elizondo, A. y Santamaría, T. (2013). Los Colegios Rurales Agrupados, primer paso al mundo docente. Contextos Educativos. Revista de Educación, 3, 315-348. Recuperado de https://publicaciones.unirioja.es/ojs/index.php/ contextos/article/view/428.

Sánchez, F., Martínez, T. y Lucena, F. (2017). Satisfacción del profesorado de la escuela rural de la provincia de Granada (España) respecto a la organización escolar. Bordón. Revista de pedagogía, 69(2), 79-96. Recuperado de https://recyt.fecyt.es/ index.php/BORDON/article/view/41372.

Santamaria, R. (2018). La Inspección ante la brecha educativa rural-urbano en España. Avances en Supervisión Educativa, 30. https://doi.org/10.23824/ase.v0i30.634.

Sepúlveda-Ruiz, M. P. y Gallardo-Gil, M. (2011). La escuela rural en la sociedad globalizada: nuevos caminos para una realidad silenciada. Profesorado, 15(2), 141-153. Recuperado de https://www.ugr.es/ recfpro/rev152ART9.pdf.

Tahull, J. y Montero, I. (2018). Reflexiones sobre la escuela rural. Un modelo educativo de éxito. Tendencias Pedagógicas, 32, 161-176. http://doi.org/10.15366/ tp2018.32.012.

Vázquez-Recio, R. (2016). Las escuelas públicas rurales: entre el bien común y la exclusión. Revista Interuniversitaria de Formación del Profesorado, 30(1), 67-79. Recuperado de http://www.aufop.com/aufop/revistas/arta/impresa/194/1997.

Vigo, B. y Soriano, J. (2015). Family involvement in creative teaching practices for all in small rural schools. Ethnography and Education, 10(3), 325-339. http://doi.org/ 10.1080/17457823.2015.1050044. 